\title{
Three-Dimensional Radiative Transfer Tomography for Turbid Media
}

\author{
W. Cai, M. Xu, and R. R. Alfano
}

\begin{abstract}
The photon distribution, as a function of position, angle, and time, is computed using the analytical cumulant solution of the Boltzmann radiative transfer equation (RTE). A linear forward model for light propagation in turbid media for three-dimensional (3-D) optical tomography is formed based on this solution. The model can be used with time resolved, continuous wave $(\mathrm{CW})$, and frequency-domain measurements in parallel geometries. This cumulant forward model (CFM) is more accurate than that based on the diffusion approximation of RTE. An inverse algorithm that incorporates this CFM is developed, based on a fast 3-D hybriddual-Fourier tomographic approach using multiple detectors and multiple sources in parallel geometries. The inverse algorithm can produce a 3-D image of a turbid medium with more than 20000 voxels in 1-2 min using a personal computer. A 3-D image reconstructed from simulated data is presented.
\end{abstract}

Index Terms-Absorption and scattering, forward model, inverse algorithm, optical tomography, photon migration, radiative transfer equation (RTE).

\section{INTRODUCTION}

$\mathbf{O}$ VER THE PAST decade, optical tomography has been investigated as a noninvasive imaging method that uses nonionizing near-infrared (NIR) light to obtain images of the interior of the breast. Unlike X-ray, which is attenuated through media by ionizing the electrons at inner-orbits of atoms, NIR light uses the vibrational overtones for different molecular components in the structures of tumor. NIR light may be used to create image based on the molecular change, which may be used to improve sensitivity and specificity in the early diagnostics of breast cancer. Breast tissues scatter light strongly, and blur the direct shadow image of a tumor. A technique, known as inverse image reconstruction, has been investigated to overcome the problem of multiple scattering. Some obstacles in the development of optical tomography are inaccuracy of the commonly used diffusion forward model, and lack of a fast inverse algorithm able to realize a three-dimensional (3-D) image reconstruction of a breast for clinical use.

One critical issue is the forward model, which should correctly simulate photon propagation in the medium. The most commonly used forward models were built based on solution

Manuscript received November 6, 2002; revised February 11, 2003. This work was supported in part by the U.S. Army Medical Research and Materiels Command under Grant DAMD17-98-1-8147, in part by NASA, and in part by the Office of Naval Research (ONR).

The authors are with the Institute for Ultrafast Spectroscopy and Lasers, New York State Center of Advanced Technology for Ultrafast Photonic Materials and Applications, Department of Physics, The City College and Graduate Center of City University of New York, New York, NY 10031 USA (e-mail: alfano@scisun.sci.ccny.cuny.edu).

Digital Object Identifier 10.1109/JSTQE.2003.813312 of the diffusion equation, which is the lowest approximation of the radiative transfer equation (RTE) [1]-[5]. The forward models based on the diffusion approximation (DA) give a large error when the distance $d$ between a voxel and a source is small. Furthermore, the photon distribution still maintains a strong anisotropy in a deeper region away from a source, which will be shown later in this paper. Unfortunately, contributions from near surface voxels to measured signals are often larger than contributions from the voxels deep inside the medium. Inaccuracy of the DA-based forward model may lead to a failure in image reconstruction, especially for small hidden objects deep inside the medium. The total weight matrix should be inverted. The large elements in the matrix, which play a more important role in inversion, are evaluated incorrectly in DA models. The shortcoming of DA is well recognized, but it is still broadly applied due to the difficulty in directly solving the radiative transfer equation. Hielscher et al. [6] and Vihunen et al. [7] developed numerical solutions of RTE for optical tomography.

Recently, we have developed an analytical solution of RTE, based on cumulant expansion, in an infinite uniform medium with an arbitrary phase function [8], [9]. It provides an explicit analytical expression for photon distribution function $I(\mathbf{r}, \mathbf{s}, t)$, as a function of position $\mathbf{r}$, direction of light $\mathbf{s}$, and time $t$. The mean position and the half-width at half-maximum (HWHM) height of the distribution are always exact. In this paper, the linear forward model based on the cumulant solution is described. This CFM may used with time-resolved, continuous wave $(\mathrm{CW})$, and frequency-domain data, which are much more accurate than the DA models.

To obtain a 3-D image one needs to investigate the inverse algorithms. For clinical applications, this requires an inversion technique, that is computationally fast, and stable in the presence of measurement noise. Recent algorithms to solve the inverse problem include Newton's least-square-based methods and gradient-descent methods [1]-[5]. These approaches use an iterative procedure, which requires a long computation time to solve a 3-D inverse problem with large unknowns (the number of unknowns is the number of voxels). Furthermore, the iterative methods can not ensure that the result arrives at a "global minimum," and does not converge to a "local minimum," which is not a true image of the medium. The application of Fourier transform, which has been called "diffraction tomography," can greatly reduce computation time. Matson et al. [10] and Li et al. [11] have developed the diffraction optical tomographic methods to realize fast image reconstruction. However, their algorithms are limited to the use of a single light source with a two-dimensional (2-D) plane 
of detectors. This type of experimental setup acquires only a set of 2-D data using $\mathrm{CW}$ or frequency-modulated light, that is not enough for a 3-D image reconstruction. Recently, Schotland and Markel developed inverse inversion algorithms using diffusion tomography [12]-[14] based on the analytical form of the Green's function of frequency-domain diffusive waves, and point-like absorbers and scatterers. Data obtained by multiple sources with multiple detectors in parallel slab geometry are used in these approaches.

A fast hybrid-dual-Fourier (HDF) algorithm, which uses multiple sources and multiple detectors in parallel slab geometry, is described in this paper for reconstruction of a 3-D image of an inhomogeneous medium. This approach uses a general 2-D translation invariance of the Green's function in a homogeneous background slab medium, suitable for forward models based on solution of RTE, and various other forward models, in CW, frequency-domain, and time-resolved measurements. This inverse algorithm runs fast. It is shown that a 3-D image of a turbid medium (for example, divided into $32 \times 32 \times 20=20480$ voxels) can be reconstructed in 1-2 min using a personal computer. This algorithm can produce stable images in presence of relatively strong noises.

The forward model and the inverse algorithm discussed in the following can also be applied for image reconstruction in a cloudy environment for military use.

This paper is organized as follows. Section II presents the analytical solution of RTE, based on a cumulant expansion, in an infinite uniform medium and shows the photon distribution function computed using the cumulant analytical solution. Section III describes the forward models based on the analytical solution of RTE, considering the slab geometry, and a weak heterogeneity using a perturbative method. Section IV describes the HDF inverse algorithm for a reconstruction of a 3-D image of an inhomogeneous medium. The 3-D image using this algorithm is shown. A discussion is presented in Section V.

\section{Analytical Cumulant Solution of RTE}

The photon propagation in a medium is described by the photon distribution function, $I(\mathbf{r}, \mathbf{s}, t)$, as a function of time $t$, position $\mathbf{r}$, and direction $\mathbf{s}$. The mathematical equation governing photon propagation is the well-known radiative transfer equation

$$
\begin{aligned}
& \partial I(\mathrm{r}, \mathrm{s}, t) / \partial t+c \mathrm{~s} \cdot \nabla_{\mathrm{r}} I(\mathrm{r}, \mathrm{s}, t)+\mu_{a}(\mathrm{r}) I(\mathrm{r}, \mathrm{s}, t) \\
& =\mu_{s}(\mathrm{r}) \int P\left(\mathrm{~s}, \mathrm{~s}^{\prime}, \mathrm{r}\right)\left[I\left(\mathrm{r}, \mathrm{s}^{\prime}, t\right)-I(\mathrm{r}, \mathrm{s}, t)\right] d \mathrm{~s}^{\prime} \\
& \quad+\delta\left(\mathrm{r}-\mathrm{r}_{0}\right) \delta\left(\mathrm{s}-\mathrm{s}_{0}\right) \delta(t-0)
\end{aligned}
$$

where the fundamental parameters are the scattering rate $\mu_{\mathrm{s}}(\mathbf{r})=c \rho \sigma_{\mathrm{s}}$, the absorption rate $\mu_{a}(\mathbf{r})=c \rho \sigma_{a}$, and the differential angular scattering rate $\mu_{\mathrm{s}}(\mathbf{r}) P\left(\mathbf{s}, \mathbf{s}^{\prime}, \mathbf{r}\right)$, where $\sigma_{a}$ and $\sigma_{\mathrm{s}}$ are the absorption and scattering cross sections respectively, $\rho$ is density of scatterers, and $c$ is the speed of light in the medium. In a uniform infinite medium, these parameters are position independent.
When the phase function depends only on the scattering angle, we can expand the phase function in Legendre polynomials with constant coefficients

$$
P\left(\mathrm{~s}, \mathrm{~s}^{\prime}\right)=\frac{1}{4 \pi} \sum_{l} a_{l} P_{l}\left[\cos \left(\mathrm{s} \cdot \mathrm{s}^{\prime}\right)\right] .
$$

Recently, we have developed a new approach to obtain an analytical solution of RTE, based on a cumulant expansion, in an infinite uniform medium, with an arbitrary phase function $P\left(\mathbf{s}, \mathbf{s}^{\prime}\right)$ [8], [9].

We briefly review the concept of "cumulant" in a one-dimensional (1-D) case. Consider a random variable $x$, with a probability distribution function $f(x)$. Instead of using $f(x)$ to describe the distribution, we define the $n$th moment of $x,\left\langle x^{n}\right\rangle=\int x^{n} f(x) d x$, and correspondingly the $n$th cumulant $\left\langle x^{n}\right\rangle_{c}$ defined by $\exp \left(\sum_{n=1}^{\infty}\left\langle x^{n}\right\rangle_{c}(i t)^{n} / n !\right)=\langle\exp (i t x)\rangle=$ $\sum_{n=0}^{\infty}\left\langle x^{n}\right\rangle(i t)^{n} / n$ !. The first cumulant $\langle x\rangle_{c}$ is the mean position of $x$. The second cumulant $\left\langle x^{2}\right\rangle_{c}$ represents the HWHM of the distribution. The higher cumulants are related to the detailed shape of the distribution. For example, $\left\langle x^{3}\right\rangle_{c}$ describes the skewness or asymmetry of the distribution, and $\left\langle x^{4}\right\rangle_{c}$ describes the "kurtosis" of the distribution, that is the extent to which it differs from the standard bell shape associated with the normal distribution function. The cumulants, hence, describe the distribution in an intrinsic way by subtracting off the effects of all lower order moments. In 3-D case, the first cumulant has three components, the second cumulant has six components, and so on.

We derived an explicit algebraic expression of spatial cumulants at any angle and any time that is exact up to an arbitrarily high order $n$ [9]. This means the distribution function $I(\mathbf{r}, \mathbf{s}, t)$ can be computed to any desired accuracy. At the second order, $n=2$, an analytic, hence, useful explicit expression for distribution function $I(\mathbf{r}, \mathbf{s}, t)$ is obtained [8]. This distribution is Gaussian in position, which is accurate at later times, but only provides the exact mean position and the exact HWHM at early times. A weakness of the second order cumulant solution is that photons at the front edge of Gaussian distribution travel faster than light speed, thus violate causality, though to a much less extent than that in the DA.

Fig. 1 compares $I(\mathbf{r}, \mathbf{s}, t)$ obtained from the analytical cumulant solution and the Monte Carlo (MC) simulation. In order to reduce the statistical deviation to an acceptable level, $10^{9}$ events are counted in the MC simulation. The figure shows that the solid curve (the tenth-order cumulant solution) is located in the middle of data obtained by the $\mathrm{MC}$ simulation. The solution for $\mathrm{CW}$ case can be obtained by an integration of $I(\mathbf{r}, \mathbf{s}, t)$ over time $t$. It is shown that even second order cumulant solution (the dotted curve) can provide an accurate $\mathrm{CW}$ solution, because this solution ensures that the mean position and the HWHM of distribution are always exact.

The plots in Fig. 1 indicate that a strong anisotropic angular distribution still exists at $z \sim 6 l_{t r}\left(l_{t r}\right.$ is the transport mean free path) from the source. The DA is only valid when the angular distribution is nearly isotropic. The dominate $s$ wave distribution $N(\mathbf{r}, t) / 4 \pi$ computed using the diffusion model (the thick dotted curve) has a large discrepancy with the MC result. 


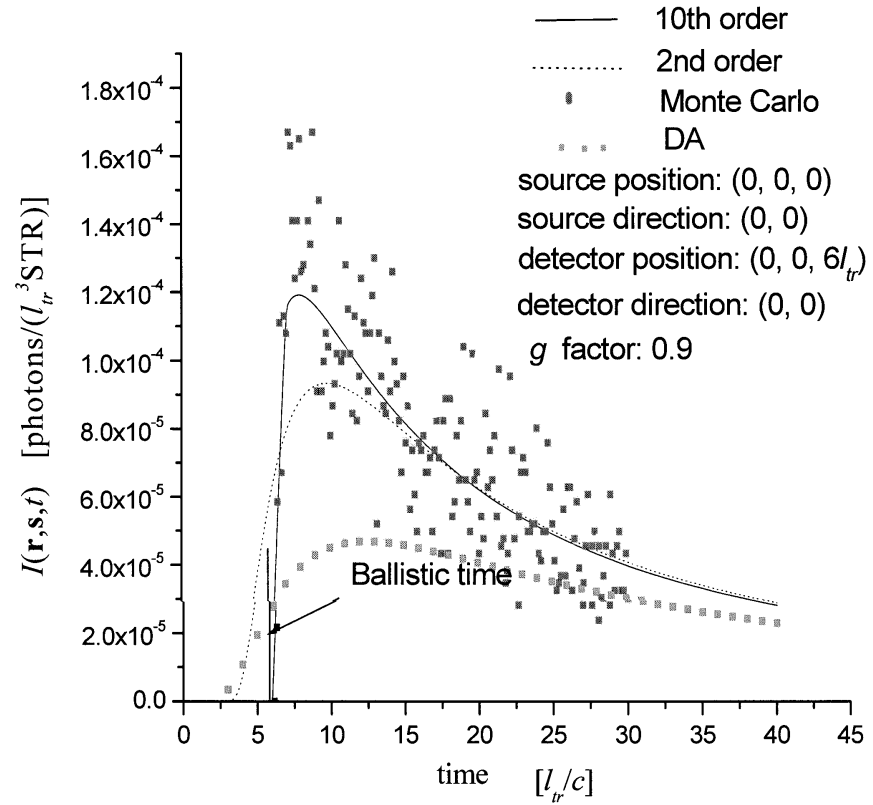

Fig. 1. Distribution function $I(\mathbf{r}, \mathbf{s}, t)$ in an infinite uniform scattering medium as a function of time $t$, using Henyey-Greenstein phase function with $g=0.9$. The detector is located at $R=6 l_{t r}=60 l_{s}$ from the source front along direction of incident light, and the direction is along the incident direction. The solid curve is computed from approximation up to tenth order of cumulant; the dotted curve is computed from approximation up to the second order of cumulant, the discrete dots are from the MC simulation; the curve of thick dots is from the DA, $N(\mathrm{r}, t) / 4 \pi$.

The second-order analytical cumulant solution is given by [8]

$$
\begin{aligned}
& I(\mathrm{r}, \mathrm{s}, t)=\frac{F\left(\mathrm{~s}, \mathrm{~s}_{0}, t\right)}{(4 \pi)^{3 / 2}} \frac{1}{(\operatorname{det} B)^{1 / 2}} \\
& \quad \cdot \exp \left[-\frac{1}{4}\left(B^{-1}\right)_{\alpha \beta}\left(r-r^{c}\right)_{\alpha}\left(r-r^{c}\right)_{\beta}\right]
\end{aligned}
$$

where

$$
\begin{aligned}
F & \left(\mathrm{~s}, \mathrm{~s}_{0}, t\right) \\
& =\exp \left(-\mu_{a} t\right) \sum_{l} \frac{2 l+1}{4 \pi} \exp \left(-g_{l} t\right) P_{l}\left[\cos \left(\mathrm{s} \cdot \mathrm{s}_{0}\right)\right] \\
& =\exp \left(-\mu_{a} t\right) \sum_{l} \frac{2 l+1}{4 \pi} \exp \left(-g_{l} t\right) \sum_{m} Y_{l m}(\mathrm{~s}) Y_{l m}^{*}\left(\mathrm{~s}_{0}\right) .
\end{aligned}
$$

In (4), $g_{l}=\mu_{\mathrm{s}}\left[1-a_{l} /(2 l+1)\right], Y_{l m}(\theta, \phi)=(-1)^{m}[(l-$ $m) ! /(l+m) !]^{1 / 2} P_{l}^{(m)}(\cos \theta) \exp (i m \phi)$, where $P_{l}^{(m)}(\cos \theta)$ is the associated Legendre function, and $Y_{l m}(\mathbf{s})$ are spherical harmonics normalized to $4 \pi /(2 l+1)$.

In (3), the mean position of the distribution (first cumulant), when the source is located at $\mathbf{r}_{0}=0$ and the incident direction is along $z$, is given by

$$
\begin{aligned}
r_{z}^{c}(\mathrm{~s}, t)= & G \sum_{l} A_{l} P_{l}(\cos \theta) \\
& \cdot\left[(l+1) f\left(g_{l}-g_{l+1}\right)+l f\left(g_{l}-g_{l-1}\right)\right] \\
r_{x}^{c}(\mathrm{~s}, t)= & G \sum_{l} A_{l} P_{l}^{(1)}(\cos \theta) \\
& \cdot \cos \phi\left[f\left(g_{l}-g_{l-1}\right)-f\left(g_{l}-g_{l+1}\right)\right]
\end{aligned}
$$

where

$$
\begin{aligned}
G & =c \exp \left(-\mu_{a} t\right) / F\left(\mathbf{s}, \mathbf{s}_{0}, t\right) \\
f(g) & =[\exp (g t)-1] / g \\
A_{l} & =(1 / 4 \pi) \exp \left(-g_{l} t\right)
\end{aligned}
$$

$r_{y}^{c}$ is obtained by replacing $\cos \phi$ in (5.2) by $\sin \phi$.

The HWHM (second cumulant) is expressed as

$$
B_{\alpha \beta}(\mathrm{s}, t)=c G \Delta_{\alpha \beta}-r_{\alpha}^{c} r_{\beta}^{c} / 2
$$

with

$$
\begin{aligned}
\Delta_{z z}= & \sum_{l} A_{l} P_{l}(\cos \theta)\left[\frac{l(l-1)}{2 l-1} E_{l}^{(1)}+\frac{(l+1)(l+2)}{2 l+3} E_{l}^{(2)}\right. \\
& \left.+\frac{l^{2}}{2 l-1} E_{l}^{(3)}+\frac{(l+1)^{2}}{2 l+3} E_{l}^{(4)}\right] \\
\Delta_{x x, y y}= & \sum_{l} \frac{1}{2} A_{l} P_{l}(\cos \theta)\left[-\frac{l(l-1)}{2 l-1} E_{l}^{(1)}\right. \\
& -\frac{(l+1)(l+2)}{2 l+3} E_{l}^{(2)}+\frac{l(l-1)}{2 l-1} E_{l}^{(3)} \\
& \left.+\frac{(l+1)(l+2)}{2 l+3} E_{l}^{(4)}\right] \pm \sum_{l} \frac{1}{2} A_{l} P_{l}^{(2)}(\cos \theta) \\
& \cdot \cos (2 \phi)\left[\frac{1}{2 l-1} E_{l}^{(1)}+\frac{1}{2 l+3} E_{l}^{(2)}\right. \\
& \left.-\frac{1}{2 l-1} E_{l}^{(3)}-\frac{1}{2 l+3} E_{l}^{(4)}\right]
\end{aligned}
$$

where (+) corresponds to $\Delta_{x x}$ and (-) corresponds to $\Delta_{y y}$.

$$
\begin{aligned}
\Delta_{x y}= & \sum_{l} \frac{1}{2} A_{l} P_{l}^{(2)}(\cos \theta) \sin (2 \phi)\left[\frac{1}{2 l-1} E_{l}^{(1)}\right. \\
& \left.+\frac{1}{2 l+3} E_{l}^{(2)}-\frac{1}{2 l-1} E_{l}^{(3)}-\frac{1}{2 l+3} E_{l}^{(4)}\right] \\
\Delta_{x z}= & \sum_{l} \frac{1}{2} A_{l} P_{l}^{(1)}(\cos \theta) \cos (\phi)\left[\frac{2(l-1)}{2 l-1} E_{l}^{(1)}\right. \\
& \left.-\frac{2(l+2)}{2 l+3} E_{l}^{(2)}+\frac{1}{2 l-1} E_{l}^{(3)}+\frac{1}{2 l+3} E_{l}^{(4)}\right] .
\end{aligned}
$$

$\Delta_{y z}$ is obtained by replacing $\cos \phi$ in (8.4) by $\sin \phi$. In (8.1)-(8.4) $E_{l}^{(1-4)}$ are given by

$$
\begin{aligned}
& E_{l}^{(1)}=\left[f\left(g_{l}-g_{l-2}\right)-f\left(g_{l}-g_{l-1}\right)\right] /\left(g_{l-1}-g_{l-2}\right) \\
& E_{l}^{(2)}=\left[f\left(g_{l}-g_{l+2}\right)-f\left(g_{l}-g_{l+1}\right)\right] /\left(g_{l+1}-g_{l+2}\right) \\
& E_{l}^{(3)}=\left[f\left(g_{l}-g_{l-1}\right)-t\right] /\left(g_{l}-g_{l-1}\right) \\
& E_{l}^{(4)}=\left[f\left(g_{l}-g_{l+1}\right)-t\right] /\left(g_{l}-g_{l+1}\right) .
\end{aligned}
$$

Fig. 2(a) and (b) shows the light distribution as a function of time at different receiving angles in an infinite uniform medium, computed by the second cumulant solution, where detector is located, separately, at $5 l_{t r}$ [Fig. 2(a)] and $15 l_{t r}$ [Fig. 2(b)] from the source in the incident direction of the source. Fig. 2 shows the existence of the strong anisotropy of the light distribution at 


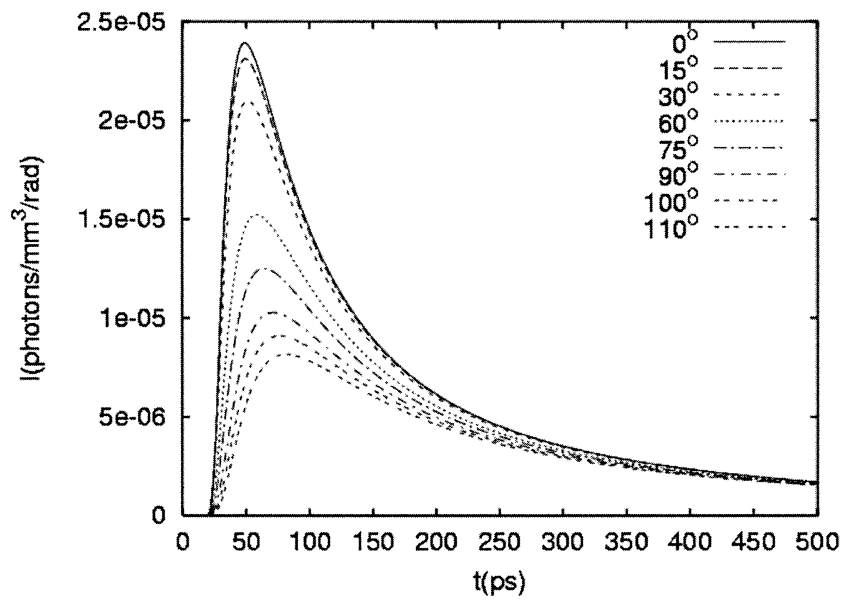

(a)

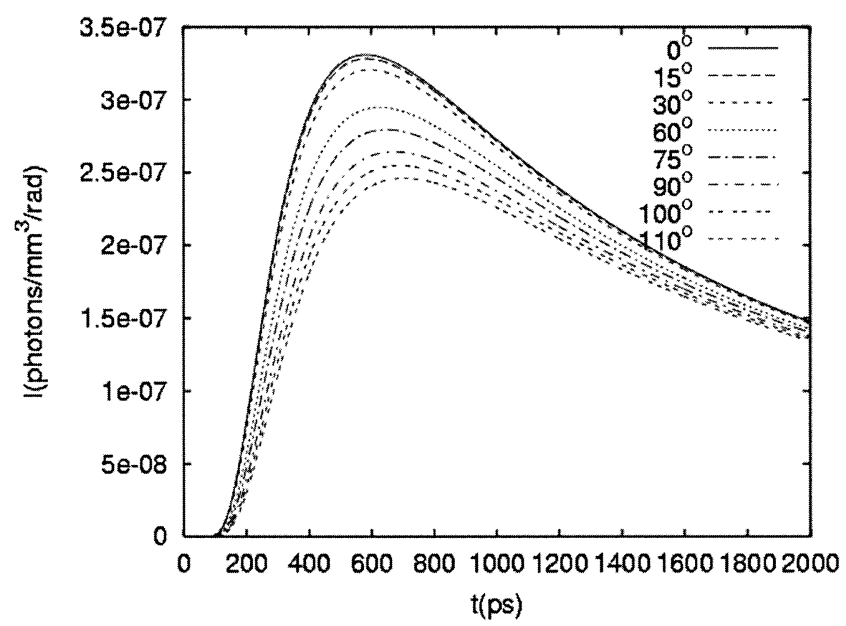

(b)

Fig. 2. Light distribution in an infinite uniform medium as a function of time at different received angle, using second cumulant solution of radiative transfer equation, where detector is located, separately, at 10 [Fig. 2(a)] and $30 \mathrm{~mm}$ [Fig. 2(b)] from the source in the incident direction. The parameters for this calculation are: $l_{t r}=2 \mathrm{~mm}, l_{a}=300 \mathrm{~mm}$, the phase function is computed using Mie theory for polystyrene spheres with diameter $d=1.11 \mu \mathrm{m}$ in water and the wavelength of laser source $\lambda=625 \mathrm{~nm}$, which gives the g-factor $g=0.926$.

$5 l_{t r}$ from the source and the modest anisotropy at a distance of $15 l_{t r}$. These types of distributions have been demonstrated by time-resolved experiments [15].

One advantage of using the above analytical solution of RTE is that the distribution function can be computed very fast. The associated Legendre functions can be accurately computed using recurrence relations. It takes only a minute to compute $10^{5}$ data of $I(\mathbf{r}, \mathbf{s}, t)$ on a personal computer.

The corresponding solution in the frequency-domain $I(\mathbf{r}, \mathbf{s}, \omega)$ can be obtained by making a Fourier transform $\int d t \exp (-i \omega t) I(\mathrm{r}, \mathrm{s}, t)$. The $\mathrm{CW}$ solution is obtained by taking $\omega=0$.

The photon density $N(\mathbf{r}, t)$ of the second cumulant solution is given by

$$
\begin{array}{r}
N(\mathrm{r}, t)=\frac{1}{\left(4 \pi D_{z z} c t\right)^{1 / 2}} \frac{1}{4 \pi D_{x x} c t} \exp \left[-\frac{\left(z-R_{z}\right)^{2}}{4 D_{z z} c t}\right] \\
\cdot \exp \left[-\frac{\left(x^{2}+y^{2}\right)}{4 D_{x x} c t}\right] \exp \left(-\mu_{a} t\right)
\end{array}
$$

with the mean position

$$
R_{z}=c\left[1-\exp \left(-g_{1} t\right)\right] / g_{1} .
$$

The corresponding time-dependent diffusion coefficients are

$$
\begin{aligned}
D_{z z}=\frac{c}{3 t}\left\{\frac{t}{g_{1}}-\frac{3 g_{1}-g_{2}}{g_{1}^{2}\left(g_{1}-g_{2}\right)}\left[1-\exp \left(-g_{1} t\right)\right]\right. \\
\quad+\frac{2}{g_{2}\left(g_{1}-g_{2}\right)}\left[1-\exp \left(-g_{2} t\right)\right] \\
\left.\quad-\frac{3}{2 g_{1}^{2}}\left[1-\exp \left(-g_{1} t\right)\right]^{2}\right\} \\
D_{x x}=D_{y y} \\
=\frac{c}{3 t}\left\{\frac{t}{g_{1}}+\frac{g_{2}}{g_{1}^{2}\left(g_{1}-g_{2}\right)}\left[1-\exp \left(-g_{1} t\right)\right]\right. \\
\left.\quad-\frac{1}{g_{2}\left(g_{1}-g_{2}\right)}\left[1-\exp \left(-g_{2} t\right)\right]\right\} .
\end{aligned}
$$

As shown in (11)-(13), the mean position of the distribution is moving, and the diffusion coefficients are time dependent. At $t \rightarrow 0$, the mean position of the photon density moves along $z$ direction with speed $c$, and the diffusion coefficients tend to zero, this result presents a clear picture of near ballistic motion. As time increases, the mean position motion slows down, and the diffusion coefficients increase from zero. This stage of photon migration is often called a snakelike mode. At long time, (10) tends to the center-moved $\left(1 l_{t r}\right)$ diffusion model with the diffusion coefficient $l_{t r} / 3$.

\section{FORWARD MODEL BASED ON THE Cumulant Solution of RTE}

The linear forward models for scattering media are built in following three steps: 1) computation of a background Green's function in an infinite uniform medium; 2) extension of this Green's function to slab geometry; and 3) computation of the weight function using a perturbative method. These steps have been applied in building the linear forward models under DA [2]. We use these steps as well, but our approach is based on the cumulant solution of RTE, rather than the solution of the diffusion equation.

We use the second-order cumulant solution for computing a background Green's function in an infinite uniform medium, since it is easy to use the explicit expressions in (3)-(9), that avoid complicated computations of higher order cumulants. The second order cumulant solution is accurate at later times, but only provides the correct mean position and the correct HWHM at early times. We notice that the width of the distribution at early times could be smaller than the size of a voxel, the average over the distributions at different points in a voxel smears the detail shape of the distribution. In the $\mathrm{CW}$ or frequency- domain cases, the shape of the distribution is further smeared by integration over time $t$. Therefore, the second-order cumulant solution can be a reasonable approximation in building forward models based on the RTE.

Since a detector usually collects emergent light within a wide range of angle of different directions, it is convenient to compute the Green's function related to a detector using photon density $N\left(\mathbf{r}_{d}, t\right)(10)-(13)$, where $\mathbf{r}_{d}$ is the position of detector. 


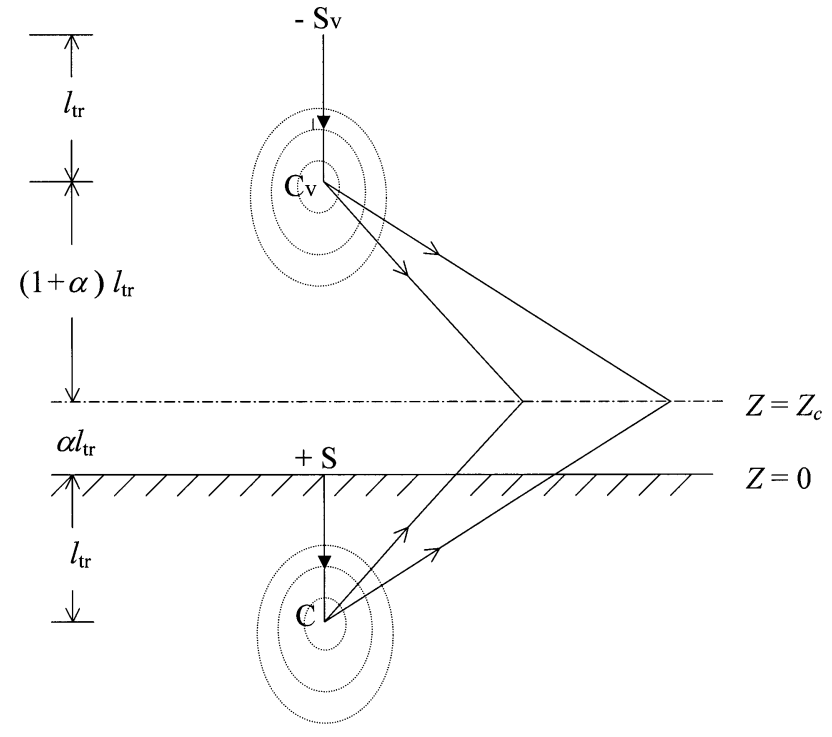

Fig. 3. Schematic diagram shows how to extend the cumulant solution of RTE from an infinite medium to a semi-infinite medium.

It is essential to include the boundary effect in the solution of the RTE when photons are injected into and spread out from a finite sized medium. A proper extension of the cumulant solution to slab geometry is an essential step for building a forward model.

A boundary condition is applied based on the following physical consideration. At early times, the center of photon distribution injected into medium, moves forward into medium. Then, the distribution spreads out from the moving center with diffusion coefficients that gradually increase from zero. At early times, the number of photons leaking out of the boundary is negligible compared to the total number of the incident photons. The boundary condition plays a role at later times, when there are many photons leaking out of the boundary.

The approach known as an approximate "extrapolated" boundary condition [16], extrapolates the boundary by a distance $\xi=\alpha l_{t r}$, the extrapolation length, beyond the real boundaries with $\alpha \sim 0.7$, at which the photon density vanishes.

To apply this boundary condition for the cumulant solution in a semi-infinite geometry a, a virtual negative source $S_{\mathrm{v}}$ is added to the original source $S$, as shown in Fig. 3. During the early period, the solution of the RTE in an infinite uniform medium automatically satisfied the boundary condition because the density is near zero at the boundary, and the virtual source does not play a role. After a time of approximately $4 l_{t r} / c$, the center of photon density, $C$, has moved and stopped at a position $1 l_{t r}$ from the original source $S$ and the center from virtual source, $C_{\mathrm{v}}$, has moved in a similar way. Then, the arrangement shown in Fig. 3, produces a cancellation of contributions to the photon density from the original source and the virtual source on the extrapolated boundary.

Fig. 4 shows that the time-resolved backscattered photon distribution in a semi-infinite medium on the $z=0$ surface, with the source-detector distance $1 l_{t r}$, obtained using the secondorder cumulant approximation and the extrapolated boundary

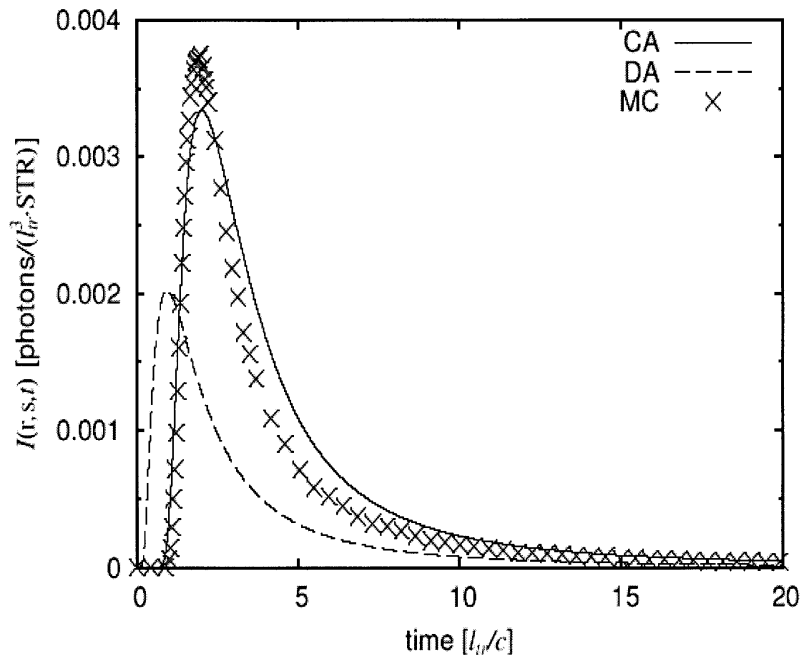

Fig. 4. Backscattered photon distribution $I(\mathbf{r}, \mathbf{s}=-\mathbf{z}, t)$ emerging from plane surface of a semi-infinite turbid medium, as a function of time, with the source-detector distance $1 l_{t r}$ on the surface $z=0$ plane. The pulse source is located at $z=0$, incident along $z$ direction. The extrapolated boundary condition is used. The solid curve is obtained from cumulant approximation (CA), up to the second cumulant. The dashed curve is from DA. The cross points are obtained from MC simulation.

condition, which agrees with the MC simulation much better than that of the DA.

For extending to the slab geometry, adding a series of pairs of virtual "image" sources at both sides of slab is a good approximation for satisfaction of the extrapolated boundary conditions on both sides of a slab [17].

The heterogeneous structure of a highly scattering turbid medium can be characterized by the following optical parameters: the scattering rate $\mu_{\mathrm{s}}(\mathbf{r})$, the absorption rate $\mu_{a}(\mathbf{r})$, and the differential angular scattering rate $\mu_{\mathbf{s}}(\mathbf{r}) P\left(\mathbf{s}, \mathbf{s}^{\prime}, \mathbf{r}\right)$.

A perturbation method is used which takes the photon distribution function in a uniform background slab medium as the zero-order approximation. The change of the photon distribution function originates from the change of optical parameters compared to that in the uniform background slab medium. The change of scattering and absorption parameters are defined as follows:

$$
\begin{aligned}
\Delta \mu_{\mathrm{s}}(\mathbf{r}) & =\mu_{\mathrm{s}}(\mathbf{r})-\mu_{\mathrm{s}}^{(0)} \\
\Delta \mu_{a}(\mathbf{r}) & =\mu_{a}(\mathbf{r})-\mu_{a}^{(0)} \\
\Delta\left[\mu_{\mathrm{s}} P\right]\left(\mathbf{s}, \mathbf{s}^{\prime}, \mathbf{r}\right) & =\mu_{\mathrm{s}}(\mathbf{r}) P\left(\mathbf{s}, \mathbf{s}^{\prime}, \mathbf{r}\right),-\mu_{\mathrm{s}}^{(0)} P^{(0)}\left(\mathbf{s}, \mathbf{s}^{\prime}\right)
\end{aligned}
$$

where the quantities with super index (0) are the optical parameters in a uniform background slab medium. By expanding $\Delta\left[\mu_{\mathrm{s}} P\right]\left(\mathbf{s}, \mathbf{s}^{\prime}, \mathbf{r}\right)$ in Legendre polynomials, we obtain

$$
\begin{aligned}
& \Delta\left[\mu_{\mathrm{s}} P\right]\left(\mathrm{s}, \mathrm{s}^{\prime}, \mathrm{r}\right) \\
& \quad=\frac{1}{4 \pi} \sum_{l}\left[\Delta \mu_{s}(\mathrm{r}) a_{l}^{(0)}+\mu_{s}^{(0)} \Delta a_{l}(\mathrm{r})\right] P_{l}\left[\cos \left(\mathrm{s} \cdot \mathrm{s}^{\prime}\right)\right]
\end{aligned}
$$

with $\Delta a_{0}(\mathbf{r})=0$, since $a_{0}$ always equals 1 . The physical meaning is that the scattering parameters have no effect on the $s(l=0)$ component. 
Making a perturbation expansion of (1) to the first-order Born approximation, the change in the photon distribution is given by

$$
\begin{aligned}
\Delta I & \left(\mathrm{r}_{\mathrm{d}}, \mathrm{s}_{\mathrm{d}}, t \mid \mathrm{r}_{\mathrm{s}}, \mathrm{s}_{\mathrm{s}}\right) \\
= & \int d t^{\prime} \int d \mathrm{r} \int d \mathrm{~s}^{\prime} I^{(0)}\left(\mathrm{r}_{\mathrm{d}}, \mathrm{s}_{\mathrm{d}}, t-t^{\prime} \mid \mathrm{r}, \mathrm{s}^{\prime}\right) \\
& \cdot\left\{\int \Delta\left[\mu_{\mathrm{s}} P\right]\left(\mathrm{s}, \mathrm{s}^{\prime}, \mathrm{r}\right) I^{(0)}\left(\mathrm{r}, \mathrm{s}, t^{\prime} \mid \mathrm{r}_{\mathrm{s}}, \mathrm{s}_{\mathrm{s}}\right) d \mathrm{~s}\right. \\
& \left.-\left[\Delta \mu_{\mathrm{s}}(\mathrm{r})+\Delta \mu_{a}(\mathrm{r})\right] I^{(0)}\left(\mathrm{r}, \mathrm{s}^{\prime}, t^{\prime} \mid \mathrm{r}_{\mathrm{s}}, \mathrm{s}_{\mathrm{s}}\right)\right\}
\end{aligned}
$$

where $\Delta I\left(\mathbf{r}_{\mathrm{d}}, \mathbf{s}_{\mathrm{d}}, t \mid \mathbf{r}_{s}, \mathbf{s}_{\mathrm{s}}\right)$ is the change in the light intensity received by a detector located at $\mathbf{r}_{\mathrm{d}}$, along the direction $\mathbf{s}_{\mathrm{d}}$, and at time $t$, which is injected from a source located at $\mathbf{r}_{\mathrm{s}}$, along a direction of $\mathbf{s}_{s}$, at time $t=0$. "Change" refers to the difference in intensity compared to that received by the same detector, from the same source, when light passes through a uniform background slab medium. The term $I^{(0)}\left(\mathbf{r}_{2}, \mathbf{s}_{2}, t \mid \mathbf{r}_{1}, \mathbf{s}_{1}\right)$ is the intensity of light, calculated using the cumulant solution of RTE, at $\mathbf{r}_{2}$ along the direction $\mathbf{s}_{2}$ and at time $t$, when light is injected from a position $\mathbf{r}_{1}$ along a direction of $\mathbf{s}_{1}$ at time $t=0$ migrating in a uniform background slab medium.

The background Green's functions in (16), obtained by cumulant solution, are expanded in spherical harmonics

$$
\begin{aligned}
I^{(0)}\left(\mathrm{r}, \mathrm{s}, t^{\prime} \mid \mathrm{r}_{\mathrm{s}}, \mathrm{s}_{\mathrm{s}}\right) & =\sum_{l, m} A_{l m}\left(\mathrm{r}, \mathrm{r}_{\mathrm{s}}, \mathrm{s}_{\mathrm{s}}, t^{\prime}\right) Y_{l m}(\mathrm{~s}), \\
I^{(0)}\left(\mathrm{r}_{\mathrm{d}}, \mathrm{s}_{\mathrm{d}}, t-t^{\prime} \mid \mathrm{r}, \mathrm{s}\right) & =\sum_{l, m} C_{l m}^{*}\left(\mathrm{r}, \mathrm{r}_{\mathrm{d}}, \mathrm{s}_{\mathrm{d}}, t-t^{\prime}\right) Y_{l m}^{*}(\mathrm{~s}) .
\end{aligned}
$$

The spherical transform is performed using a fast Fourier transform for the integral over $\phi$, and a Clenshaw-Curtis quadrature for the integral over $\theta$.

Using the orthogonality relation of the spherical function and the addition theorem: $\sum_{m} Y_{l m}(\mathrm{~s}) Y_{l m}^{*}\left(\mathrm{~s}^{\prime}\right)=P_{l}\left[\cos \left(\mathrm{s} \cdot \mathrm{s}^{\prime}\right)\right]$, the analytical integration over $\mathbf{s}$ and $\mathbf{s}^{\prime}$ in (16) can be performed. For time resolved data, the contribution from an absorbing object located at $\mathbf{r}_{k}$ is given by

$$
\begin{array}{rl}
\Delta I & I\left(\mathrm{r}_{\mathrm{d}}, \mathrm{s}_{\mathrm{d}}, \mathrm{r}_{\mathrm{s}}, \mathrm{s}_{\mathrm{s}}, t \mid \mathrm{r}_{k}\right) \\
= & -\Delta \mu_{a}\left(\mathrm{r}_{k}\right) \delta V_{k} \int_{0}^{t} d t^{\prime} \sum_{l=0}^{L} \frac{4 \pi}{(2 l+1)} \\
& \cdot \sum_{m} A_{l m}\left(\mathrm{r}_{k}, \mathrm{r}_{\mathrm{s}}, \mathrm{s}_{\mathrm{s}}, t^{\prime}\right) C_{l m}^{*}\left(\mathrm{r}_{k}, \mathrm{r}_{\mathrm{d}}, \mathrm{s}_{\mathrm{d}}, t-t^{\prime}\right)
\end{array}
$$

where $\delta V_{k}$ is the volume of $k$ th voxel, and $L$ is the cutoff value in the Legendre expansion in (18). The contribution from a scattering object located at $\mathbf{r}_{k}$ is given by

$$
\begin{aligned}
\Delta I\left(\mathrm{r}_{\mathrm{d}}, \mathrm{s}_{\mathrm{d}}, \mathrm{r}_{\mathrm{s}}, \mathrm{s}_{\mathrm{s}}, t \mid \mathrm{r}_{k}\right) \\
=-\delta V_{k} \int_{0}^{t} d t^{\prime} \sum_{l=1}^{L} \frac{4 \pi}{(2 l+1)} \\
\quad \cdot\left[\Delta \mu_{s}\left(\mathrm{r}_{k}\right)\left(1-\frac{a_{l}^{(0)}}{2 l+1}\right)-\mu_{s}^{(0)} \frac{\Delta a_{l}\left(\mathrm{r}_{k}\right)}{2 l+1}\right] \\
\quad \cdot \sum_{m} A_{l m}\left(\mathrm{r}_{k}, \mathrm{r}_{\mathrm{s}}, \mathrm{s}_{\mathrm{s}}, t^{\prime}\right) C_{l m}^{*}\left(\mathrm{r}_{k}, \mathrm{r}_{\mathrm{d}}, \mathrm{s}_{\mathrm{d}}, t-t^{\prime}\right) .
\end{aligned}
$$

For frequency domain (or CW) data, the contribution from an absorbing object located at $\mathbf{r}_{k}$ is given by

$$
\begin{array}{rl}
\Delta I & I\left(\mathrm{r}_{\mathrm{d}}, \mathrm{s}_{\mathrm{d}}, \mathrm{r}_{\mathrm{s}}, \mathrm{s}_{\mathrm{s}}, \omega \mid \mathrm{r}_{k}\right) \\
= & -\Delta \mu_{a}\left(\mathrm{r}_{k}\right) \delta V_{k} \sum_{l=0}^{L} \frac{4 \pi}{2 l+1} \\
& \cdot \sum_{m} A_{l m}\left(\mathrm{r}_{k}, \mathrm{r}_{\mathrm{s}}, \mathrm{s}_{\mathrm{s}}, \omega\right) c_{l m}^{*}\left(\mathrm{r}_{k}, \mathrm{r}_{\mathrm{d}}, \mathrm{s}_{\mathrm{d}}, \omega\right)
\end{array}
$$

and the contribution from a scattering object located at $\mathbf{r}_{k}$ is given by

$$
\begin{aligned}
\Delta I\left(\mathrm{r}_{\mathrm{d}}, \mathrm{s}_{\mathrm{d}}, \mathrm{r}_{\mathrm{s}}, \mathrm{s}_{\mathrm{s}}, \omega \mid \mathrm{r}_{k}\right) \\
=-\delta V_{k} \sum_{l=1}^{L} \frac{4 \pi}{2 l+1} \\
\cdot\left[\Delta \mu_{s}\left(\mathrm{r}_{\mathrm{k}}\right)\left(1-\frac{a_{l}^{(0)}}{2 l+1}\right)-\mu_{s}^{(0)} \frac{\Delta a_{l}\left(\mathrm{r}_{k}\right)}{2 l+1}\right] \\
\cdot \sum_{m} A_{l m}\left(\mathrm{r}_{k}, \mathrm{r}_{\mathrm{s}}, \mathrm{s}_{\mathrm{s}}, \omega\right) c_{l m}^{*}\left(\mathrm{r}_{k}, \mathrm{r}_{\mathrm{d}}, \mathrm{s}_{\mathrm{d}}, \omega\right) .
\end{aligned}
$$

Comparing (18)-(21) with the corresponding weight function commonly used in the DA, [1], [2] only $s$ wave $(l=0)$ for absorptive objects, and only $p$ wave $(l=1)$ for scattering objects are considered in the diffusion forward models. Besides, even for $s$ wave and $p$ wave, the diffusive solution is incorrect when voxels are located near the source, as discussed before.

The previous formulae allow simulating the background Green's function and the change of optical parameters in detail. They are also applicable to the cases where only a few parameters of the medium are known, similar to that for the diffusion forward model. When only $\mu_{\mathrm{s}}^{(0)}, \mu_{a}^{(0)}$, and $g$-factor for an uniform background medium are given, the Henyey-Greenstein phase function [18] is widely adopted as an approximate phase function

$$
\begin{aligned}
P(\cos \theta) & =\frac{1}{4 \pi} \frac{1-g^{2}}{\left(1+g^{2}-2 g \cos \theta\right)^{3 / 2}} \\
& =\frac{1}{4 \pi} \sum_{l}(2 l+1) g^{l} P_{l}(\cos \theta) .
\end{aligned}
$$

Although (22) uses a single parameter $g$-factor to describe a phase function, this description is much better than that used in the DA, which implies a phase function linear in $\cos \theta$.

If $\Delta a_{l}(\mathbf{r})$ in (21), which represent the change of the phase function, is not considered, two optical parameters being imaged are $\Delta \mu_{a}(\mathbf{r})$ and $\Delta \mu_{\mathrm{s}}(\mathbf{r})$. The reduced scattering coefficient $\Delta \mu_{\mathrm{s}}\left(1-a_{1}^{(0)} / 3\right)$ is directly related to $\Delta D$ (change of the diffusion coefficient) used in the DA models. The CFM, hence, can be applied to the experimental data in a similar fashion as that for the DA models, to obtain images of the optical parameters. In the CFM, however, all contributions from higher spherical waves are properly included.

The most time consuming part in computation of CFM using the previous formulae is to build a database of $A_{l m}$ and $C_{l m}^{*}$. Once it is built for a uniform background medium, the database can be applied for imaging of various heterogeneity cases. In parallel geometry, $A_{l m}$ is a function of $\left(x_{k}-x_{s}, y_{k}-y_{s}\right)$ due to the 2-D translation invariance. Since position of source $z_{s}$ and 
incident direction $\mathbf{s}_{\mathrm{s}}$ are fixed, only a 3-D $\left(x_{k}-x_{s}, y_{k}-y_{s}, z_{k}\right)$ database is required. When $\mathbf{s}_{\mathbf{s}}$ is taken along $z$ direction (light is injected perpendicular to surface), the scale of database is reduced to 2-D due to the $z$ axis symmetry. Photons from different directions in a wide solid angle are received by a detector, as discussed before, photon density $N\left(\mathbf{r}_{d}-\mathbf{r}, \mathbf{s}, t\right)$ is used for computing the Green's function associated with detectors, which is independent of $\mathbf{s}_{d}$, and $C_{l m}^{*}$ can be computed much easily. The database can be built in a reasonable computation time because the distribution function $I^{(0)}\left(\mathbf{r}_{2}, \mathbf{s}_{2}, t \mid \mathbf{r}_{1}, \mathbf{s}_{1}\right)$ can be rapidly calculated using the analytical expressions.

\section{FAST 3-D HDF INVERSE ALGORITHM}

We now outline an inverse algorithm to quickly reconstruct image of a medium from acquired measurements using the above CFM. The model, neglecting the irrelevant parameters, can be briefly written as

$$
\begin{aligned}
& Y\left(\overrightarrow{\mathrm{r}}_{\mathrm{d}}, \overrightarrow{\mathrm{r}}_{\mathrm{s}}, z_{d}, z_{s}\right) \\
& \quad=\int d \overrightarrow{\mathrm{r}} d z W\left(\overrightarrow{\mathrm{r}}_{\mathrm{d}}-\overrightarrow{\mathrm{r}}, \overrightarrow{\mathrm{r}}_{\mathrm{s}}-\overrightarrow{\mathrm{r}}, z, z_{d}, z_{s}\right) X(\overrightarrow{\mathrm{r}}, z)
\end{aligned}
$$

where $\vec{R}=(\vec{r}, \mathrm{z})$ is the position of a voxel inside turbid medium; $\overrightarrow{\mathrm{r}}$ is $(x, y)$ coordinates; $\overrightarrow{\mathrm{R}}_{\mathrm{s}}=\left(\overrightarrow{\mathrm{r}}_{\mathrm{s}}, \mathrm{z}_{\mathrm{s}}\right)$ is the position of a source; and $\overrightarrow{\mathrm{R}}_{\mathrm{d}}=\left(\overrightarrow{\mathrm{r}}_{\mathrm{d}}, \mathrm{z}_{d}\right)$ is the position of a detector. In (23), $Y\left(\overrightarrow{\mathrm{r}}_{\mathrm{d}}, \overrightarrow{\mathrm{r}}_{\mathrm{s}}, z_{d}, z_{s}\right)$ is the measured change in light intensity received by a detector at $\vec{R}_{d}$ from a point source at $\vec{R}_{\mathrm{s}}$. $X(\overrightarrow{\mathrm{r}}, z)$ is the change of the optical parameters inside turbid medium. The weight function $W\left(\vec{r}_{\mathrm{d}}-\overrightarrow{\mathrm{r}}, \overrightarrow{\mathrm{r}}_{\mathrm{s}}-\overrightarrow{\mathrm{r}}, z, z_{d}, z_{s}\right)$ is a function of $\vec{r}_{d}-\vec{r}$ and $\vec{r}_{s}-\vec{r}$ on $(x, y)$ plane, because of parallel geometry, assuming an infinite sized area, and the 2-D translation invariance of the Green's function in a background homogeneous slab. Here, the special form of the weight function is not relevant; the weight function can be calculated by the CFM or the DA models, using with $\mathrm{CW}$, frequency, or time-resolved data. This approach is general and can also be used for inverse problems of nonoptical measurements in parallel geometries.

A light source scans through a 2-D array. Transmitted or backscattered light signals emerging from the medium are detected using a 2-D array of detectors, such as a charge-coupled device (CCD) camera (or time-gated CCD camera in the time resolved case). Each illumination of the light source provides a set of 2-D data on the 2-D detector array. For CW or frequency-modulated light source, this arrangement can produce a set of $2-\mathrm{D} \times 2-\mathrm{D}=4-\mathrm{D}$ data in a relatively short acquisition time, because a CCD camera produces 2-D data of the detectors at different positions simultaneously. When time-resolved or modulation at multiple frequencies are applied, a set of five-dimensional (5-D) data can be acquired. The inverse problems of 3-D imaging, hence, are over-determined, which is necessary for obtaining an accurate 3-D image.

When the translation invariance is satisfied, the Fourier transform approach is a powerful technique to achieve a fast inversion. In the Fourier space, the convolution of $W$ and $X$ becomes a product of $W$ and $X$, and the weight matrix $W$ becomes diagonal. Hence, inversion can be performed much faster. Using this concept in the case of multiple sources and multiple detectors in parallel geometries a dual 2-D Fourier transform $\int d \overrightarrow{\mathrm{r}}_{\mathrm{s}} d \overrightarrow{\mathrm{r}}_{\mathrm{d}} e^{i \overrightarrow{\mathrm{q}}_{\mathrm{s}} \overrightarrow{\mathrm{r}}_{\mathrm{s}}} e^{i \overrightarrow{\mathrm{q}} \mathrm{d}_{\mathrm{r}} \overrightarrow{\mathrm{r}}_{\mathrm{d}}}$ is performed on (23), to obtain

$$
\begin{aligned}
\int d \overrightarrow{\mathrm{r}}_{\mathrm{s}} d \overrightarrow{\mathrm{r}}_{\mathrm{d}} e^{i \overrightarrow{\mathrm{q}}_{\mathrm{s}} \overrightarrow{\mathrm{r}}_{\mathrm{s}}} e^{i \overrightarrow{\mathrm{q}}_{\mathrm{d}} \overrightarrow{\mathrm{r}}_{\mathrm{d}}} Y\left(\overrightarrow{\mathrm{r}}_{\mathrm{s}}, \overrightarrow{\mathrm{r}}_{\mathrm{d}}, z_{s}, z_{d}\right) \\
=\int d z \int d \overrightarrow{\mathrm{r}} \int d\left(\overrightarrow{\mathrm{r}}_{\mathrm{s}}-\overrightarrow{\mathrm{r}}\right) d\left(\overrightarrow{\mathrm{r}}_{\mathrm{d}}-\overrightarrow{\mathrm{r}}\right) e^{i \overrightarrow{\mathrm{q}}_{\mathrm{s}}\left(\overrightarrow{\mathrm{r}}_{\mathrm{s}}-\overrightarrow{\mathrm{r}}\right)} e^{i \overrightarrow{\mathrm{q}}_{\mathrm{d}}\left(\overrightarrow{\mathrm{r}}_{\mathrm{d}}-\overrightarrow{\mathrm{r}}\right)} \\
\quad \cdot W\left(\overrightarrow{\mathrm{r}}_{\mathrm{s}}-\overrightarrow{\mathrm{r}}, \overrightarrow{\mathrm{r}}_{\mathrm{d}}-\overrightarrow{\mathrm{r}}, z_{s}, z_{\mathrm{d}}, z\right) e^{i\left(\overrightarrow{\mathrm{q}}_{\mathrm{s}}+\overrightarrow{\mathrm{q}}_{\mathrm{d}}\right) \overrightarrow{\mathrm{r}}} X(\overrightarrow{\mathrm{r}}, z)
\end{aligned}
$$

which leads to

$\hat{Y}\left(\overrightarrow{\mathrm{q}}_{\mathrm{d}}, \overrightarrow{\mathrm{q}}_{\mathrm{s}}, z_{d}, z_{s}\right)=\int d z \hat{W}\left(\overrightarrow{\mathrm{q}}_{\mathrm{d}}, \overrightarrow{\mathrm{q}}_{\mathrm{s}}, z, z_{d}, z_{s}\right) \hat{X}\left(\overrightarrow{\mathrm{q}}_{\mathrm{d}}+\overrightarrow{\mathrm{q}}_{\mathrm{s}}, z\right)$

where $\hat{Y}, \hat{X}$, and $\hat{W}$ are change in light intensity, change in optical parameters, and the weight function in the Fourier space, respectively.

A similar form of this dual Fourier transform has been derived by Markel and Schotland [13], [14] in a frequency-domain diffusion model.

Equation (24) seems difficult to be used for performing the inverse reconstruction because of the argument mismatch $\left(\vec{q}_{\mathrm{d}}+\right.$ $\left.\overrightarrow{\mathrm{q}}_{\mathrm{s}}\right)$ in $\hat{X}$ and $\left(\overrightarrow{\mathrm{q}}_{\mathrm{s}}, \overrightarrow{\mathrm{q}}_{\mathrm{d}}\right)$ in $\hat{Y}$ and $\hat{W}$. This difficulty occurs because the weight function in (23) is related to three positions: $\vec{r}_{d}, \vec{r}_{s}$, and $\vec{r}$. To remove this complexity, the following linear hybrid transform is introduced:

$$
\begin{aligned}
& \overrightarrow{\mathrm{u}}=\overrightarrow{\mathrm{q}}_{\mathrm{d}}+\overrightarrow{\mathrm{q}}_{\mathrm{s}} \\
& \overrightarrow{\mathrm{v}}=\overrightarrow{\mathrm{q}}_{\mathrm{d}}-\overrightarrow{\mathrm{q}}_{\mathrm{s}} .
\end{aligned}
$$

This results in the HDF formula

$$
\tilde{Y}\left(\overrightarrow{\mathrm{u}}, \overrightarrow{\mathrm{v}}, z_{d}, z_{s}\right)=\int d z \tilde{W}\left(\overrightarrow{\mathrm{u}}, \overrightarrow{\mathrm{v}}, z, z_{d}, z_{s}\right) \tilde{X}(\overrightarrow{\mathrm{u}}, z)
$$

where $\tilde{Y}, \tilde{X}$, and $\tilde{W}$ are, respectively, $\hat{Y}, \hat{X}$, and $\hat{W}$ as functions of $\vec{u}$ and $\vec{v}$.

While (25) is a relatively simple expression, it is essential to properly realize this hybrid transform in discrete lattices of the Fourier space. A procedure to quickly perform this transform from $\left(\mathrm{q}_{\mathrm{d}}, \mathrm{q}_{\mathrm{s}}\right)$ coordinates to new $(\mathrm{u}, \mathrm{v})$ coordinates, separately, for $x$ and $y$ components, is explained in Fig. 5 using an example of a $6 \times 6$ lattice. The maximum value of $u$ is taken as the maximum value of $q_{d}$ or $q_{s}$, not the maximum value of $q_{d}+q_{s}$. The periodic property of lattice in the Fourier space is used, for example, $\tilde{Y}(\mathrm{u}=2, \mathrm{v}=4)=\hat{Y}\left(\mathrm{q}_{\mathrm{s}}=3\right.$, $\left.\mathrm{q}_{\mathrm{d}}=5\right)$. This procedure builds a one-to-one correspondence between lattices in the two coordinate systems. Fig. 5 shows that $\tilde{Y}$ and $\tilde{W}$ at each node [circle in Fig. 5] in $(u, v)$ coordinates are directly mapped from $\hat{Y}$ and $\hat{W}$, respectively, at the corresponding node in $\left(\mathrm{q}_{d}, \mathrm{q}_{s}\right)$ coordinates without any algebraic manipulation.

In (26), a common 2-D Fourier argument $\vec{u}$ appears in $\tilde{Y}, \tilde{X}$, and $\tilde{W}$. For each value of $\overrightarrow{\mathrm{u}}$, (26) leads to an over-determined 1-D problem for inverse reconstruction: $\tilde{Y}(\overrightarrow{\mathrm{v}})=\int d z \tilde{W}(\overrightarrow{\mathrm{v}}, z) \tilde{X}(z)$. In order to perform fast inversion, we invert the normal form of the forward model: $\tilde{Y} \tilde{W}^{T}=\left[\tilde{W}^{T} \tilde{W}\right] \tilde{X}$ for each $\overrightarrow{\mathrm{u}}$, where $\left[\tilde{W}^{T} \tilde{W}\right]$ is a $M \times M$ matrix, with $M$ the number of layers in $z$ direction. The original $W$ in (23) is a matrix with a large dimension. The inverse 


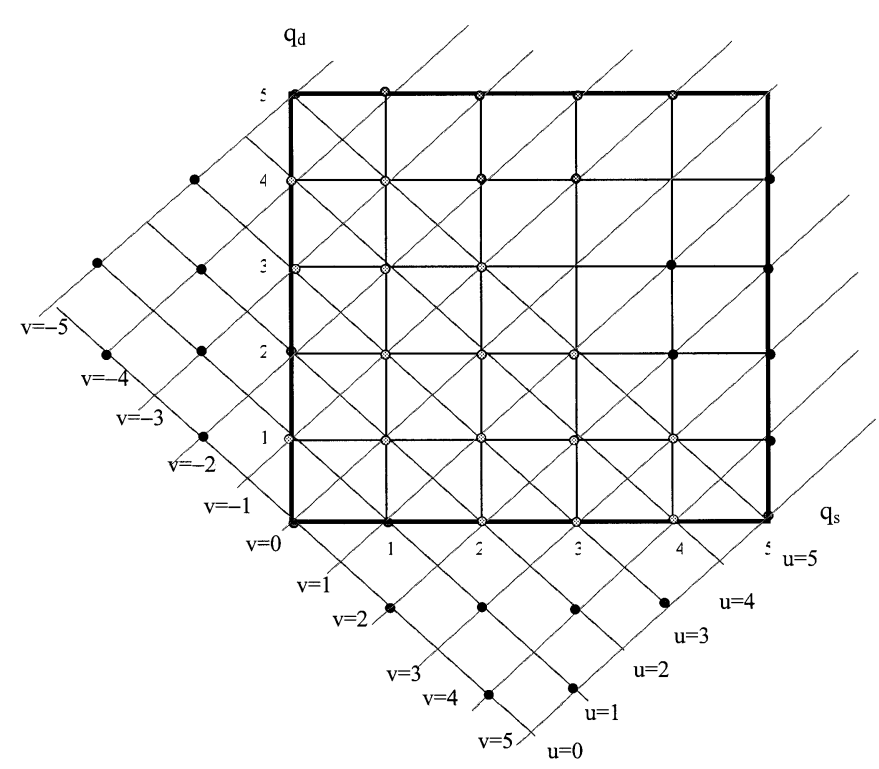

Fig. 5. Example of a $6 \times 6$ lattice for explaining the linear hybrid transform from $\left(\mathrm{q}_{\mathrm{d}}, \mathrm{q}_{\mathrm{s}}\right)$ coordinates to $(\mathrm{u}, \mathrm{v})$ coordinates.

problem now is simplified to invert many (number of discrete value of $\vec{u}$ ) matrices, each with a small dimension $M$. The latter problem is much more computationally efficient compared to the original problem of (23). Once $\tilde{X}(\overrightarrow{\mathrm{u}}, z)$ are obtained for all $\overrightarrow{\mathrm{u}}$, a 2-D inverse Fourier transform produces $X(\overrightarrow{\mathrm{r}}, z)$, which is the 3-D image of optical parameters of the medium. Markel and Schotland use different procedures for inversion. In [13], a Fourier-Laplace inversion is applied, hence, an analytic continuation of measured data to the complex plane is required for the inverse Laplace transform. In [14], an inverse procedure is performed in an argument space, similar to variables $\vec{v}$ here. Since $\vec{v}$ include 2-D variables, inversion in $\vec{v}$ space could take longer time than that of inversion in $z$ space.

As discussed previously, matrices $\tilde{W}$ and $\left[\tilde{W}^{T} \tilde{W}\right]$ for each $\overrightarrow{\mathrm{u}}$ can be calculated in advance for a uniform background slab medium. Assuming that a group of experimental data has been acquired, the following steps are taken to produce a 3-D image of the medium:

1) obtain "change" of intensities, $Y\left(\overrightarrow{\mathrm{r}}_{\mathrm{d}}, \overrightarrow{\mathrm{r}}_{\mathrm{s}}, z_{d}, z_{s}\right)$, by subtracting the intensity for a uniform background medium from the measured intensity;

2) extend the $(x, y)$ area and padding zeros, to overcome the wraparound problem in discrete convolutions [19];

3) perform a dual 2-D fast Fourier transform (FFT) of $Y\left(\overrightarrow{\mathrm{r}}_{\mathrm{d}}, \overrightarrow{\mathrm{r}}_{\mathrm{s}}, z_{d}, z_{s}\right)$ in the extended area to produce $\hat{Y}\left(\overrightarrow{\mathrm{q}}_{\mathrm{d}}, \overrightarrow{\mathrm{q}}_{\mathrm{s}}, z_{d}, z_{s}\right)$;

4) determine $\tilde{Y}\left(\overrightarrow{\mathrm{u}}, \overrightarrow{\mathrm{v}}, z_{d}, z_{s}\right)$ for each $\overrightarrow{\mathrm{u}}$, using a mapping procedure explained in Fig. 5;

5) invert $\tilde{Y} \tilde{W}^{T}=\left[\tilde{W}^{T} \tilde{W}\right] \tilde{X}$ for each $\overrightarrow{\mathrm{u}}$, which is an inverse problem involving a $M \times M$ matrix, with $M$ the number of layers along $z$ direction. Proper regularization according to noise level needs to be taken into account. Regularization will be discussed later in the paper;

6) perform an inverse 2-D FFT on $\tilde{X}(\overrightarrow{\mathrm{u}}, z)$ to produce $X(\overrightarrow{\mathrm{r}}, z)$.

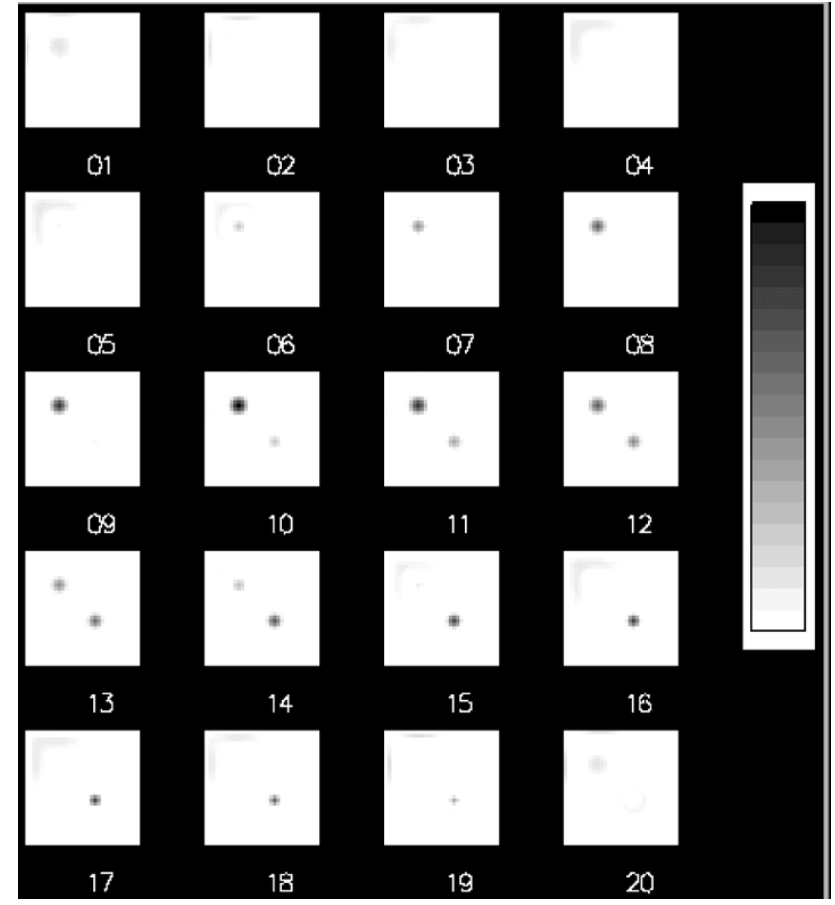

Fig. 6. 3-D image reconstructed using hybrid dual Fourier tomography. Two absorbing objects, each with the volume $3 \times 3 \times 2 \mathrm{~mm}^{3}$, are located inside a turbid medium with volume $96 \times 96 \times 40 \mathrm{~mm}^{3}$ divided into $32 \times 32 \times 20$ voxels. The first one is located at position labeled $(10,10,10)$ with absorption difference $\Delta \mu_{a}=0.01 \mathrm{~mm}^{-1}$. The second one is located at position labeled $(20,20,15)$ with absorption difference $\Delta \mu_{a}=0.007 \mathrm{~mm}^{-1}$. A CW light source incident perpendicular to the $z_{s}=0$ plane is scanned through a $2 \mathrm{D} 32 \times$ 32 array at the plane, with each pixel $3 \mathrm{~mm} \times 3 \mathrm{~mm}$. A same sized 2D array of detectors is located at $z_{d}$ plane (transmission geometry). The simulated data are produced with noise $5 \%$. A linear scale of color bar from the maximum value to minimum value of $\Delta \mu_{a}$ is used. The numbers labels the $z$ layers counting form source to the detector, layers are separated by $2 \mathrm{~mm}$.

Our computational experiments show it takes only 1-2 min on a personal computer to perform an inverse reconstruction of a 3-D image of a medium with a large number of voxels (for example, $32 \times 32 \times 20$ voxels) using this HDF algorithm.

To demonstrate our concept of HDF tomography in 3-D image reconstruction, an example using simulated $\mathrm{CW}$ data is presented. A slab turbid medium, with a transport mean-free path $l_{t r}=1 \mathrm{~mm}$, absorption length $l_{a}=300 \mathrm{~mm}$, and thickness $z_{d}=40 \mathrm{~mm}$, is divided into 20 layers. A CW light source, injected perpendicular to the $z_{s}=0$ plane, scans by a 2-D $32 \times 32$ array on the plane, with each pixel $3 \times 3 \mathrm{~mm}$. A 2-D array of detectors with the same spacing is located at $z_{d}$ plane (transmission geometry). The medium, is divided into $32 \times 32 \times 20$ voxels, each of dimension $3 \times 3 \times 2 \mathrm{~mm}^{3}$. Two absorbing objects are located in the medium, each with a volume $3 \times 3 \times 2 \mathrm{~mm}^{3}$. The first one located at $(10,10,10)$ has an absorption difference of $\Delta \mu_{a}=0.01 \mathrm{~mm}^{-1}$ with the background. The second one is located at $(20,20,15)$ with an absorption difference of $0.007 \mathrm{~mm}^{-1}$. The simulated data with noise level of 5\% are obtained using the CFM. The tomographic images are shown in Fig. 6. As shown, the central positions of 3 -D image of the objects are correct, located at a voxel $(10,10$, $10)$ with dark color, and a voxel $(20,20,15)$ with gray level. The resolution of image is about $\sim 6 \mathrm{~mm}$ in the transverse 
(x, y) plane and $\sim 10 \mathrm{~mm}$ along $z$ direction. In general, the axial resolution (along $z$ direction) is poorer than the lateral resolution [on the $(x, y)$ plane]. In transmission geometry, two Green's functions in the weight function compensate each other when the $z$ position of the object changes, that leads to a poor sensitivity of the measured photon intensity to the $z$ position of the object. The shapes of 3-D image of two objects are ellipsoids with longer axis along the $z$ direction. The absorption difference has the maximum value at the center of ellipsoid, and decays gradually with increase distance from the center.

A cutoff in discrete lattices of $\vec{q}_{s}$ and $\vec{q}_{d}$ naturally introduces a kind of regularization. This regularization is very effective. Initial tests show that even adding $30 \%$ of fluctuations on simulated data of $Y\left(\vec{r}_{\mathrm{d}}, \overrightarrow{\mathrm{r}}_{\mathrm{s}}, z_{d}, z_{s}\right)$, an image similar to that shown in Fig. 6 is still reconstructed. The reason for this is that noises come from fluctuations at different source and detector positions, which are mainly the high-frequency components of $\vec{q}_{s}$ and $\overrightarrow{\mathrm{q}}_{\mathrm{d}}$. A cutoff in $\overrightarrow{\mathrm{q}}_{\mathrm{s}}$ and $\overrightarrow{\mathrm{q}}_{\mathrm{d}}$ naturally eliminates these high frequency noises, such that a stable image, especially in $(x, y)$ plane, can be reconstructed in a strong noise level.

However, the inverse problem is still ill-posed, because contribution to the change of intensity from a small voxel deeply inside medium is weak, and is not sensitive to its $z$ position in transmission case. A regularization procedure on inversion of $\tilde{Y} \tilde{W}^{T}=\left[\tilde{W}^{T} \tilde{W}\right] \tilde{X}$ is still needed. The standard Tikhonov regularization approach [20] is applied and L-curve [21], [22] method is used for determining the best regularization parameters.

This fast inverse algorithm produces a 3-D image in a linear image regime. For nonlinear image reconstruction procedure, the reconstructed 3-D image provides a good initial profile for further refining the 3-D image taking the nonlinear effects into consideration.

The HDF inversion method can be extended to a cylindrical geometry, with an arbitrary shape of the $(x, y)$ cross section, for 3-D image reconstruction. In this geometry, an algorithm using a single Fourier inversion has been developed [23]. This algorithm is limited to the case that the sources and the detectors are located on a plane with same $z$ coordinates. The hybrid-dualFourier inverse approach in cylindrical geometry removes this restriction, so more data can be acquired for 3-D tomography. The linear forward model in cylindrical geometry is given by

$Y\left(\overrightarrow{\mathrm{r}}_{\mathrm{d}}, \overrightarrow{\mathrm{r}}_{\mathrm{s}}, z_{d}, z_{s}\right)$

$$
=\int d \overrightarrow{\mathrm{r}} d z W\left(\overrightarrow{\mathrm{r}}_{\mathrm{d}}, \overrightarrow{\mathrm{r}}_{\mathrm{s}}, \overrightarrow{\mathrm{r}} ; z_{d}-z, z_{s}-z\right) X(\overrightarrow{\mathrm{r}}, z)
$$

where $W\left(\overrightarrow{\mathrm{r}}_{\mathrm{d}}, \overrightarrow{\mathrm{r}}_{\mathrm{s}}, \overrightarrow{\mathrm{r}} ; z_{d}-z, z_{s}-z\right)$ is the weight function, a function of $\mathrm{z}_{\mathrm{d}}-z$ and $\mathrm{z}_{\mathrm{S}}-z$ due to the 1-D translation invariance of the Green's function in a homogeneous background medium in cylindrical geometry (assuming infinite $z$ length). We make a dual 1-D (along $z$ direction) Fourier transform $\int d z_{d} d z_{s} e^{i q_{d} z_{d}} e^{i q_{s} z_{s}}$ on (27) to obtain

$\hat{Y}\left(\mathrm{q}_{\mathrm{d}}, \mathrm{q}_{\mathrm{s}}, \overrightarrow{\mathrm{r}}_{d}, \overrightarrow{\mathrm{r}}_{s}\right)=\int d z \hat{W}\left(\mathrm{q}_{\mathrm{d}}, \mathrm{q}_{\mathrm{s}}, \overrightarrow{\mathrm{r}}, \overrightarrow{\mathrm{r}}_{d}, \overrightarrow{\mathrm{r}}_{s}\right) \hat{X}\left(\mathrm{q}_{\mathrm{d}}+\mathrm{q}_{\mathrm{s}}, \overrightarrow{\mathrm{r}}\right)$

where $\hat{Y}, \hat{X}$, and $\hat{W}$ are the Fourier space quantities corresponding that in (27).
The (1-D) linear hybrid coordinate transforms, $u=q_{d}+q_{s}$, and $\mathrm{v}=\mathrm{q}_{\mathrm{d}}-\mathrm{q}_{\mathrm{s}}$, for (28) leads to

$$
\tilde{Y}\left(\mathrm{u}, \mathrm{v}, \overrightarrow{\mathrm{r}}_{d}, \overrightarrow{\mathrm{r}}_{s}\right)=\int d \overrightarrow{\mathrm{r}} \tilde{W}\left(\mathrm{u}, \mathrm{v}, \overrightarrow{\mathrm{r}}_{d}, \overrightarrow{\mathrm{r}}_{s} ; \overrightarrow{\mathrm{r}}\right) \tilde{X}(\mathrm{u}, \overrightarrow{\mathrm{r}})
$$

where $\tilde{Y}, \tilde{X}$, and $\tilde{W}$ are, respectively, $\hat{Y}, \hat{X}$, and $\hat{W}$ as functions of $u$ and $v$. For each value of $u,(29)$ is an over determined 2-D problem for inverse reconstruction, namely, to determine a 2-D unknown value of $\tilde{X}(\mathrm{u}, \overrightarrow{\mathrm{r}})$ from known 3-D data of $\tilde{Y}\left(\mathrm{u}, \mathrm{v}, \overrightarrow{\mathrm{r}}_{\mathrm{d}}, \overrightarrow{\mathrm{r}}_{\mathrm{s}}\right)$ for each $\mathrm{u}$. This 3-D-2-D determination enhances the accuracy of 3-D image compared to 2-D-2-D determination in the single-Fourier transform inversion. After $\tilde{X}(\mathrm{u}, \overrightarrow{\mathrm{r}})$ are obtained for all $\mathrm{u}$, a 1-D inverse Fourier transform produces the image $X(\overrightarrow{\mathrm{r}}, z)$.

\section{DISCUSSION}

As shown in (19) and (21), there is no contribution from $s$ wave to the weight function for a scattering object. This result reflects a fact that no scattering effect exists for an isotropic angular distribution. In the regions far from sources, the weight function contributed from scattering objects is small because there is no contribution from the dominant $s$ wave, as shown in many results based on the diffusion models [1]-[5]. This nonsensitivity of signals to the scattering objects deep inside the medium should be considered in optical tomography. A pure isotropic distribution is never achieved, otherwise, there will be no flux in any directions. In the diffusive model, a small $p$ wave, $-(3 / 4 \pi) D \mathrm{~s} \cdot \nabla N$, exists which maintains the photons diffusing to the regions with fewer photons. The factor $-\nabla N$ represents this effect. However, this expression is valid only in the regions where the $p$ wave is much smaller than $s$ wave, $(1 / 4 \pi) N$, and does not correctly describe the early photon propagation near sources. Since only the weight function for scattering objects close to sources plays an important role, but it was estimated using the formula valid in regions far from sources, substantial error introduced in the diffusion forward model for scattering objects is crucial, considering $\mu_{s}^{\prime} \gg \mu_{a}$ in tissue.

For the weight function of absorbing objects, contributions from all spherical components, including $s$ wave, are given in (18) and (20). In commonly used diffusion formula, the contribution from $p$ wave was neglected. The diffusion coefficient originally derived in the DA is $D=1 /\left(3 \mu_{s}^{\prime}+\mu_{a}\right)$, that leads to $\Delta D=-D^{(0) 2}\left(3 \Delta \mu_{s}^{\prime}+\Delta \mu_{a}\right)$. The contribution from $p$ wave to the weight function for absorbing objects, hence, should exist. However, in the later diffusion models, $\Delta D$ is assigned only for scattering objects and only $s$ wave for absorbing objects is taken. Equations (18) and (20) provide a quantitative estimation of weight function for absorbing objects in regions close to the source, as well as far from the source.

The CFM and the HDF inverse algorithm need further improvements in the following aspects. Further improvement should be considered without significantly increasing complexity in computation. First, the second cumulant solution is not accurate in the detailed shape of the distribution, especially, the front edge in the Gaussian distribution violates causality. An empirical distribution, which keeps the exact value of the first and second cumulants, while satisfies the causality, can be designed to replace the Gaussian distribution. 
Second, the boundary condition is approximate. When a more accurate distribution $I(\mathbf{r}, \mathbf{s}, t)$ at early time is needed, the boundary condition for a semi-infinite geometry should be

$$
I(x, y, z=0 ; \theta, \phi, t)=0, \quad \text { if } \cos \theta>0 .
$$

This type of the boundary condition was studied by Domke [24] for the steady state case. The solution is represented as a superposition of a solution describing a transport problem in an infinite medium, and a Fredholm integral term, which corrects this solution for the appropriate half-space boundary condition. This approach may be used for further development of the boundary problem.

Third, to consider the nonlinear effects, $I^{(0)} \mathrm{s}$ in (16) should be replaced by the Green's function in a real heterogeneous medium. Among the high-order perturbative corrections of the Green's function, the "self-energy" diagram, which counts photon round trips through a position up to infinite times, plays an important role. Gandjbakhche et al. [25] studied this effect using a random walk model. We find that a renormalization procedure for this nonlinear effect can be performed after image is obtained using a linear inversion process. This renormalization procedure can recover the optimal value of the optical parameters and can improve the resolution of image. The detailed results of the renormalization will be published elsewhere.

The translation invariance is valid for the parallel geometry assuming that the $(x, y)$ area is infinite. We suppose that this assumption of the infinite area is reasonable. How much error arises due to the finite area of a sample will be studied in details.

Use of the simulated data mainly tests the validity of the inverse algorithm, does not test accuracy of the forward model. Experimental data from phantoms and in vivo measurements in human body will be performed for further testing of our approach.

\section{ACKNOWLEDGMENT}

The authors would like to thank S. K. Gayen for carefully reading this manuscript and for useful discussions.

\section{REFERENCES}

[1] A. G. Yodh, B. Tromberg, E. Sevick-Muraca, and D. Pine, Eds., "Diffusion photons in turbid media," in Special Issue J. Opt. Soc. A., 1997, vol. 14 , pp. $136-342$.

[2] S. R. Arridge, "Optical tomography in medical imaging," Inv. Probl., vol. 15 , pp. 41-93, 1999.

[3] N. Iftimia and H. Jiang, "Quantitative optical image reconstruction of turbid media using de measurements," Appl. Opt., vol. 39, pp. 5256-5261, 2000.

[4] B. W. Pogue, S. P. Poplack, T. O. McBride, W. A. Wells, U. L. Osterberg, and K. D. Paulsen, "Quantitative hemoglobin tomography with diffuse near infrared spectroscopy: Pilot results in the breast," Radiology, vol. 218, pp. 261-266, 2001.
[5] J. C. Hebden, H. Veenstra, H. Dehghani, E. M. C. Hillman, M. Schweiger, S. R. Arridge, and D. T. Delpy, "Three dimensional time-resolved optical tomography of a conical breast phantom," Appl. Opt., vol. 40, pp. 3278-3287, 2001.

[6] H. Hielscher, R. E. Alcouffe, and B. L. Barbour, "Comparison of finitedifference transport and diffusion calculation for photon migration in homogeneous and heterogeneous tissues," Phys. Med. Biol., vol. 43, pp. 1285-1302, 1998.

[7] T. Vilhunen, M. Vanhkonen, V. Kolehmainen, and J. P. Kaipio, "Linking the radiative transfer equation and the diffusion approximation," in Proc. Dig. OSA Biomedical Topical Meeting, 2002, pp. 12-14.

[8] W. Cai, M. Lax, and R. R. Alfano, "Cumulant solution of the elastic Boltzmann transport equation in an infinite uniform medium," Phys. Rev. E, Stat. Phys. Plasmas Fluids Relat. Interdiscip. Top., vol. 61, pp. 3871-3876, 2000.

[9] _ _ "Analytical solution of the elastic Boltzmann transport equation in an infinite uniform medium using cumulant expansion," J. Phys. Chem., vol. B104, pp. 3996-4000, 2000.

[10] C. L. Matson, N. Clark, L. McMackin, and J. S. Fender, "Three-dimensional tumor localization in thick tissue with the use of diffuse photondensity waves," Appl. Opt., vol. 36, pp. 214-220, 1997.

[11] X. D. Li, T. Durduran, A. G. Yodh, B. Chance, and D. N. Pattanayak, "Diffraction tomography for biochemical imaging with diffuse-photon density waves," Opt. Lett., vol. 22, pp. 573-575, 1997.

[12] J. C. Schotland and V. A. Markel, "Inverse scattering with diffusing wave," J. Opt. Soc. Amer. A, vol. 18, pp. 2767-2777, 2001.

[13] V. A. Markel and J. C. Schotland, "Inverse problem in optical diffusion tomography. I Fourier-Laplace inversion formulas," J. Opt. Soc. Amer. A, vol. 18, pp. 1336-1347, 2001.

[14] —, "Inverse scattering for the diffusion equation with general boundary conditions," Phys. Rev. E, Stat. Phys. Plasmas Fluids Relat. Interdiscip. Top., vol. 64035 601, pp. 1-4, 2001.

[15] M. E. Zevallos, A. Ya Polischuck, B. B. Das, F. Liu, and R. R. Alfano, "Time-resolved photon-scattering measurements from scattering media fitted to noneuclidean and conventional diffusion models," Phys. Rev. E, Stat. Phys. Plasmas Fluids Relat. Interdiscip. Top., vol. 57, pp. 7244-7253, 1998.

[16] M. S. Patterson, B. Chance, and B. C. Wilson, "Time-resolved reflectance and transmittance for the noninvasive measurement of tissue optical properties," Appl. Opt., vol. 28, pp. 2331-2336, 1989.

[17] M. Xu, W. Cai, M. Lax, and R. R. Alfano, "Photon migration in turbid media using a cumulant approximation to radiative transfer," Phys. Rev. E, Stat. Phys. Plasmas Fluids Relat. Interdiscip. Top., vol. 651066609 , pp. 1-10, 2002.

[18] L. G. Henyey and J. L. Greenstein, "Diffuse radiation in the galaxy," Astrophys. J., vol. 93, pp. 70-83, 1941.

[19] W. H. Press, B. P. Flannery, S. A. Teukolsky, and W. T. Vetterling, $N u-$ merical Recipes. Cambridge, U.K.: Cambridge Univ. Press, 1986, pp. 407-412.

[20] A. N. Tikhonov and A. V. Groncharsky, Eds., Ill-Posed Problems in the Natural Sciences. Moscow, USSR.: MIR, 1987.

[21] P. Hansen and D. O'Leavy, "The use of the L-curve in the regularization of discrete ill-posed problems," SIAM J. Sci. Comput., vol. 14, pp. 1487-1503, 1993.

[22] W. Cai, S. K. Gayen, M. Xu, M. Zevallos, M. Alrubaiee, M. Lax, and R. R. Alfano, "Optical tomographic image reconstruction from ultrafast time-sliced transmission measurements," Appl. Opt., vol. 38, pp. 4237-4246, 1999.

[23] W. Cai, B. B. Das, F. Liu, F. A. Zeng, M. Lax, and R. R. Alfano, "Three dimensional image reconstruction in highly scattering turbid media," in Proc. SPIE, 1997, paper 2979, pp. 241-248.

[24] H. Domke, "Reduction of radiative transfer problems in semi-infinite media to linear Fredholm integral equations," J. Quant. Spectrosc. Radiat. Trans., vol. 16, pp. 973-981, 1976.

[25] A. H. Gandjbakhche, R. F. Bonner, R. Nossal, and G. H. Weiss, "Absorptivity contrast in transillumination imaging of tissue abnormalities," Appl. Opt., vol. 35, pp. 1767-1774, 1996. 